\title{
EP-46
}

\section{Laparoscopic radical and conservative surgery of hydatid liver echinococcosis. PSM based comparative analysis of immediate and long-term outcomes}

\author{
Natalia ELIZAROVA*, Mikhail EFANOV, Ruslan ALIKHANOV, Olga MELEKHINA, Yulia KULEZNEVA, Victor TSVIRKUN \\ Department of Hepatopancreatobiliary Surgery, Loginov Moscow Clinical Scientific Center, Russia
}

Introduction: The diffusion of laparoscopic radical surgery of hydatid liver echinococcosis remains limited. There no published data on a comparative analysis of the immediate and long-term results of radical and conservative laparoscopic surgery for liver hydatid cysts. Comparison of the immediate and long-term outcomes after laparoscopic radical and conservative cystectomies was aimed.

Methods: HPB center (center 1) and general surgery hospital in endemic area (center 2) participated in retrospective study. Radical surgery included total, subtotal pericystectomy and liver resection. Conservative surgery comprised cystectomy without/with partial pericystectomy.

Results: The total number of patients was 204. Laparoscopic cystectomy was performed in 97 (47\%) patients. Radical (n-40) and conservative (n-61) laparoscopic procedures were performed in center 1 and center 2, respectively. Twenty-five pairs of patients were matched. The length of hospital stay and time of abdominal drainage were significantly shorter after radical surgery before and after PSM. The rate of severe morbidity did not differ. The mean follow-up length was 34 and 45 months in center 1 and center 2 , respectively. No differences were found in the disease recurrence rate.

Conclusions: Laparoscopic radical surgery leads to a reduction in the treatment time and can be recommended as the preferred treatment option in a specialized HPB center. 